\title{
Líquidos lónicos: Aplicações e Perspectivas Futuras
}

\author{
Luís C. Branco \\ LAQV, REQUIMTE, Departamento de Química, Faculdade de Ciências e Tecnologia, Universidade Nova de Lisboa \\ l.branco@fct.unl.pt
}

\begin{abstract}
Ionic Liquids: Applications and Future Perspectives - In last three decades, Ionic Liquids have been largely studied as relevant research topic for application in different scientific areas. The peculiar properties of ionic liquids as well as the need to develop greener chemical processes contribute for the enormous impact of this class of organic salts in the scientific community. This article describes some relevant applications and future perspectives of task-specific ionic liquids in organic chemistry, catalysis, biocatalysis, pharmaceutical chemistry, analytical chemistry, electrochemistry, chemical engineering, biotechnology and material science.
\end{abstract}

$\mathrm{O}_{\mathrm{s}}$ Líquidos Iónicos surgiram nas últimas três décadas como um tópico de investigação de elevado interesse em diferentes áreas científicas. As suas propriedades peculiares e uma necessidade da comunidade científica em desenvolver processos quimicos mais sustentáveis contribuiram para um impulso significativo desta classe de sais orgânicos. Neste artigo estão enquadradas algumas das aplicações mais relevantes dos líquidos iónicos e suas perspectivas futuras em química orgânica, catálise, biocatálise, química farmacêutica, química analítica, electroquímica, engenharia química, biotecnologia e ciência dos materiais.

\section{INTRODUÇÃO}

Os Líquidos Iónicos (LIs) são geralmente definidos como uma classe de sais orgânicos com um ponto de fusão inferior a $100^{\circ} \mathrm{C}$. Geralmente, eles possuem na sua composição uma combinação de catiões orgânicos de baixa simetria e uma variedade de aniões orgânicos e inorgânicos. O número de possiveis combinações catião-anião é muito elevado, estima-se que superior a $10^{12}$. Esta característica tem sido largamente explorada no desenvolvimento de novos LIs que possam incorporar na sua estrutura diferentes propriedades de acordo com a aplicação desejada [1].

Uma das razões do elevado interesse por parte da comunidade científica nesta classe de sais orgânicos baseia-se nas suas propriedades peculiares, em especial a sua muito reduzida pressão de vapor (quase inexistente), elevada estabilidade térmica e química, elevada condutividade iónica, facilidade de dissolução de materiais orgânicos, inorgânicos e poliméricos e uma larga janela electroquímica. Uma das características mais relevantes dos LIs relaciona-se com a possibilidade de se conseguir modelar diferentes propriedades físicas, térmicas e químicas de acordo com a combinação adequada do catião e do anião.

Apesar do enorme crescimento da área dos Líquidos Iónicos nas últimas três décadas, é possível descobrir referências a sais orgânicos de baixo ponto de fusão enquadrados na definição de líquido iónico desde o início do século Xx. Nesse contexto, uma das primeiras referências surgiu em 1914 por P. Walden reportando um sal de nitrato de etilamónio com um ponto de fusão de $12^{\circ} \mathrm{C}$ [2] e mais tarde em 1951 por Hurley e colaboradores apresentando sais de cloroaluminatos de n-alquilpiridínio de baixo ponto de fu- são [3]. Desde 1980, diferentes LIs têm sido desenvolvidos preferencialmente baseados em catiões orgânicos do tipo imidazólio, piridínio, pirrolidínio, amónio, fosfónio, sulfónio, guanidinio, tiazólio, triazólio, entre outros com diversos substituintes [4].

Os aniões podem ser escolhidos de acordo com as propriedades finais, modulando muitas vezes a viscosidade, solubilidade, polaridade, densidade, ponto de fusão e estabilidade (química e térmica) do líquido iónico final.

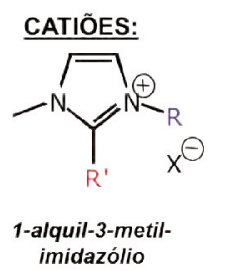

ANIÕ
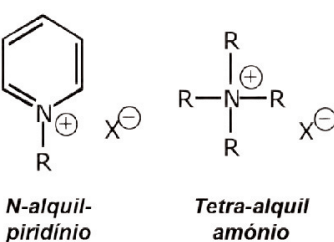

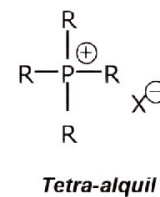

fosfónio

Figura 1 - Estruturas mais comuns de catiões orgânicos e aniões orgânicos ou inorgânicos constituintes dos Liquidos Iónicos

A evolução dos Líquidos Iónicos nas três últimas décadas pode ser dividida em três gerações de acordo com o potencial e interesse das suas aplicações finais: numa primeira fase os LIs foram preparados tendo por base o seu uso como solventes alternativos aos solventes orgânicos convencionais (voláteis e tóxicos) para inúmeros processos quimicos; numa segunda fase utilizando os LIs como materiais avançados para aplicações específicas nas áreas de engenharia química e da ciência dos materiais e numa fase mais recente associando uma componente biológica com potencial aplicação na área da bioquímica e da farmacêutica. 
A comunidade científica portuguesa tem tido um papel de relevo na área dos Líquidos Iónicos quer em termos de síntese e caracterização de novos LIs, estudos das suas propriedades fisico-químicas bem como a aplicação de LIs em diferentes áreas científicas tais como catálise, biocatálise, processos de separação e extracção, química farmacêutica, biotecnologia e no desenvolvimento de diversos materiais funcionais. Nesse contexto, deve ser realçado o trabalho do grupo de investigação do Prof. Luis Paulo Rebelo que em colaboração com grupos do Reino Unido e Estados Unidos demonstraram que existem LIs que podem ser destilados desde que se utilizem pressões de forma controlada [5].

Esta observação pode permitir, se necessário, uma purificação adicional de alguns líquidos iónicos apliando o seu campo de aplicação.

Apesar do enorme crescimento da área dos Líquidos Iónicos em termos académicos, o qual pode ser bem evidenciado no seu elevado número de publicações nas últimas décadas, as aplicações a nível industrial ainda são reduzidas.

O interesse da indústria na área dos Líquidos Iónicos ainda se encontra numa fase de crescimento mas existem algumas aplicações que têm sido exploradas [6]. Nesse particular será de destacar o processo BASIL da empresa alemã BASF que permite a obtenção de cloreto de 1-metilimidazólio em quantidades industriais [7]. Este processo tem por base a produção de alcoxifenilfosfinas (precursor de um fotoiniciador), em que a empresa substituiu trietilamina usada no processo original por 1-metilimidazolo como um agente sequestrador do ácido que se forma no decorrer da reacção. Esta optimização do processo químico permitiu facilitar a purificação do produto final, bem como a obtenção de um líquido iónico puro numa escala de algumas toneladas.

Na figura 2 apresentam-se algumas das áreas onde os LIs têm tido uma aplicação mais relevante e que iremos descrever de uma forma mais detalhada.

\section{Aplicações em SINTESE ORGÂNICA E (BIO)CATÁLISE}

Ao longo dos últimos anos diversos grupos têm investigado a potencialidade dos LIs como um meio reaccional alternativo de diversas reacções orgânicas por simples substituição do solvente orgânico convencional. Nesse contexto, inúmeras reacções químicas que envolvem formação de ligações do tipo carbono-carbono têm sido largamente exploradas em LIs tais como reacções de condensação aldólica, Friedel-Crafts, Diels-Alder, Baylis-Hilman, Wittig, alquilações entre outras [8]. Em paralelo, reacções que envolvem formação de ligações do tipo carbono com nitrogénio, oxigénio, enxofre e halogéneos também têm sido descritas [8]. Em geral, os LIs podem ser excelentes meios alternativos aos solventes orgânicos convencionais permitindo tempos reaccionais menores, facilidade de purificação e rendimentos químicos superiores. Uma das grandes potencialidades dos LIs e com maior número de estudos publicados enquadra-se no seu uso em reacções catalíticas homogéneas e heterogéneas $[9,10]$. Os catalisadores de metais de transição podem ser dissolvidos e estabilizados na fase de líquido iónico com vantagens significativas em termos de actividade catalítica e possibilidade de re-utilização para um elevado número de ciclos reaccionais. A possibilidade de reciclagem eficiente do meio reaccional constituido por LI e catalisador metálico pode estar associada ao processo de extracção do produto no final de cada reacção [11]. Em muitos processos catalíticos tem sido descrita a utilização de solventes orgânicos (éteres e alcanos) que possam dissolver o produto final e não dissolver o LI e catalisador, como um método preferencial [11]. A descoberta da solubilidade muito reduzida dos LIs em $\mathrm{CO}_{2}$ supercrítico e por outro lado a elevada solubilidade do $\mathrm{CO}_{2}$ em LIs, permite a utilização deste fluído supercrítico como um processo mais sustentável para extracção eficiente dos produtos [12].

Outras abordagens que têm sido descritas na literatura em processos de catálise envolvem o desenvolvimento de LIs suportados com a incorporação do catalisador na unidade catiónica ou aniónica [13] e de LIs quirais com interesse para reacções assimétricas [14,15].

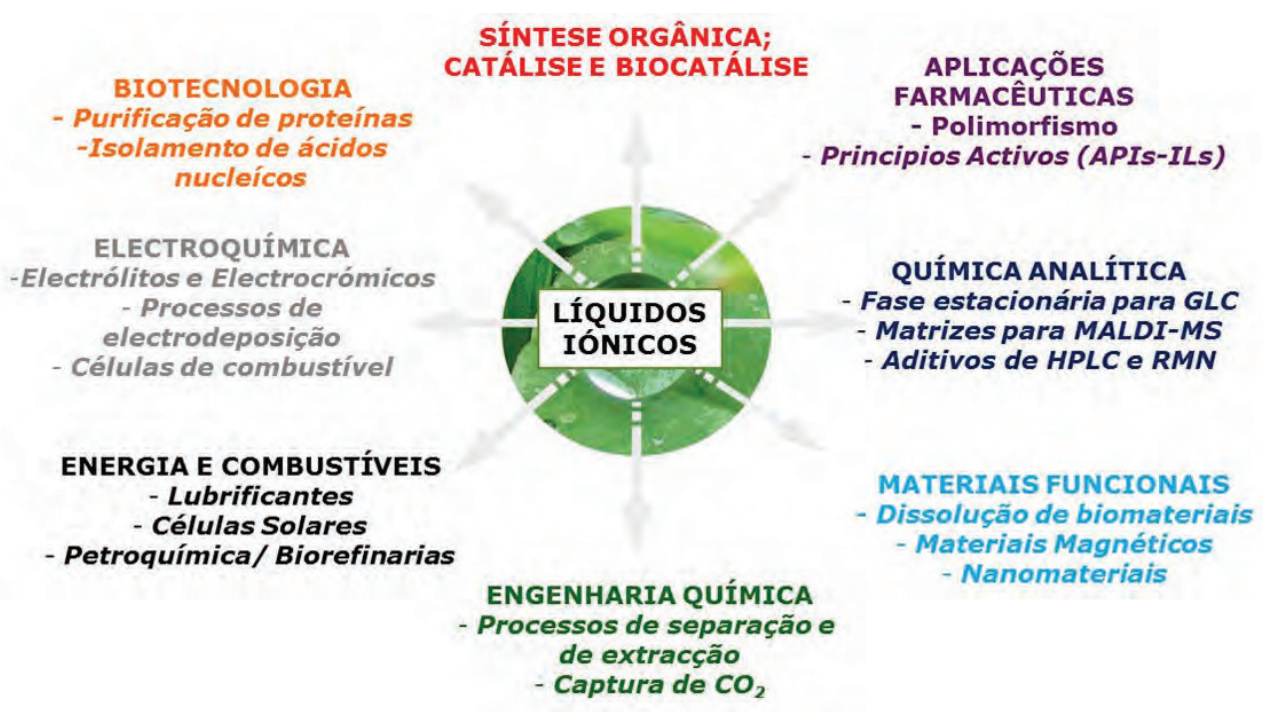

Figura 2 - Algumas das possiveis aplicações dos Líquidos Iónicos 
Os LIs quirais têm sido reconhecidos como tendo elevado potencial para processos de discriminação quiral incluindo em síntese assimétrica e na resolução de racematos [16]. Diferentes LIs quirais têm sido aplicados como aditivos quirais de modo a induzir enantiosseletividade moderadas a elevadas preferencialmente em reacções assimétricas de fotoisomerização, adição de Michael, Diels-Alder, Baylis-Hilman e di-hidroxilação de olefinas, entre outras $[17,18]$. Mais recentemente, têm sido descritos diferentes tipos de LIs quirais através da introdução de unidades quirais no catião orgânico ou através do uso de unidades quirais naturais em especial aminoácidos, açúcares e ácidos carboxílicos ou sulfónicos quirais [19].

A potencialidade do uso de LIs quirais como meio reaccional quiral (meio reaccional único ou em quantidade catalítica dissolvidos em LIs convencionais) combinados com a extracção do produto com $\mathrm{CO}_{2}$ supercrítico deverá ser um processo sustentável a ser explorado nos próximos anos com enorme potencial de aplicação industrial.

Em processos de biocatálise, o uso de LIs tem sido muito estudado como co-solvente ou solvente puro devido à sua elevada capacidade de estabilização de diferentes sistemas enzimáticos [20]. Nesse contexto, diferentes classes de enzimas tais como hidrolases (proteases e lipases) e oxidorredutases (peroxidases e desidrogenases) podem ser suspensas em LIs com um aumento da solubilidade de diversos substratos, maior actividade catalítica, rendimentos químicos elevados e minimização de reacção secundárias [21]. As vantagens do uso de LIs em biocatálise ficam demonstradas em reacções de transesterificação catalisadas pela enzima protease quimiotripsina e especialmente com lipase Candida antártica de tipo B (CAL B) e ainda em processos de resolução cinética de álcoois e derivados de aminoácidos [22,23].

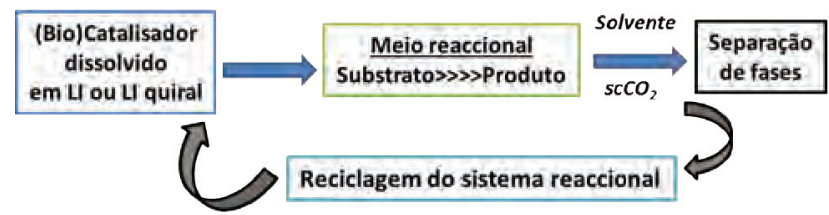

Figura 3 - Processo de reacção e posterior isolamento do produto final e reciclagem do sistema reaccional com (bio)catalisador

\section{APLICAÇÕES EM QUÍMICA FARMACÊUTICA}

Os LIs têm sido explorados em diversas aplicações farmacêuticas tais como: uso como solventes alternativos na síntese de alguns fármacos; em processos de extracção de compostos farmacêuticos de soluções aquosas; em processos que envolvam a distribuição controlada de fármacos e mais recentemente através do desenvolvimento de LIs farmacêuticos (designados API-ILs) [24].

Algumas publicações recentes têm descrito o uso de LIs como meio reaccional para a síntese de nucleosídeos baseados em fármacos antivirais (brivudine, stavudine e trifluridine) com rendimentos, graus de pureza e tempos re- accionais significativamente melhores que em sistemas de solventes convencionais [24].

Outros exemplos relacionam-se com a síntese de compostos híbridos de aplicação antiparasitária, compostos com potencial aplicação antitumoral (por exemplo borofenilalanina; ésteres derivados do ácido cafeico) e anti-inflamatórios não-esteróides (exemplo do pravadoline e do (S)-naproxeno) [24].

O uso dos LIs em aplicações farmacêuticas tem suscitado debate científico alargado em relação à sua toxicidade e biodegradabilidade, e apesar do crescente número de estudos biológicos de diferentes tipos de LIs, os resultados não são totalmente conclusivos [25,26]. No entanto, a possibilidade de preparar LIs de reduzida toxicidade estará dependente da selecção de catiões e aniões não tóxicos e biocompatíveis [27].

Em geral será possível concluir que muitos dos excipientes farmacêuticos convencionais tais como dimetilssulfóxido e surfactantes não-iónicos possuem valores de toxicidade semelhantes aos observados para diversos LIs [25]. No caso dos LIs deve ainda ser referida a potencial vantagem adicional de possuirem elevada biodegradabilidade por comparação com outros excipientes.

A sua aplicação está ainda limitada apesar da sua elevada capacidade de dissolução e estabilização de fármacos e puderem ser excelentes transportadores de fármacos de reduzida solubilidade em água.

Um dos grandes problemas da indústria farmacêutica prende-se com o polimorfismo de muitos fármacos ou seja a sua tendência para cristalizarem com subsequente redução da actividade terapêutica [28]. A indústria farmacêutica tem feito um investimento significativo na procura de soluções para este problema através do uso de co-cristais, formas amorfas e fármacos imobilizados em polímeros, mas muitas destas estratégias não apresentam resultados positivos. Nesse contexto, a transformação de princípios activos normalmente em estado sólido à temperatura ambiente em líquidos ou sólidos de baixo ponto de fusão pode solucionar o problema do polimorfismo [28].

A preparação de novos LIs farmacêuticos ou a simples dissolução de fármacos em LIs biocompatíveis pode ter no futuro um impacto relevante na indústria farmacêutica com a respectiva eliminação do polimorfismo bem como um aumento da biodisponibilidade e actividade terapêutica [29].

Desde 2008 diferentes grupos de investigação têm desenvolvido LIs farmacêuticos (API-ILs) baseados em diferentes classes de fármacos [30,31]. Das várias contribuições para esta área devem ser referidos alguns contributos portugueses em especial para o desenvolvimento de novos LIs farmacêuticos baseados em antibióticos (ampicilina, ciprofloxacina e norfloxacina) utilizando processos sintéticos mais sustentáveis [32,33]. 


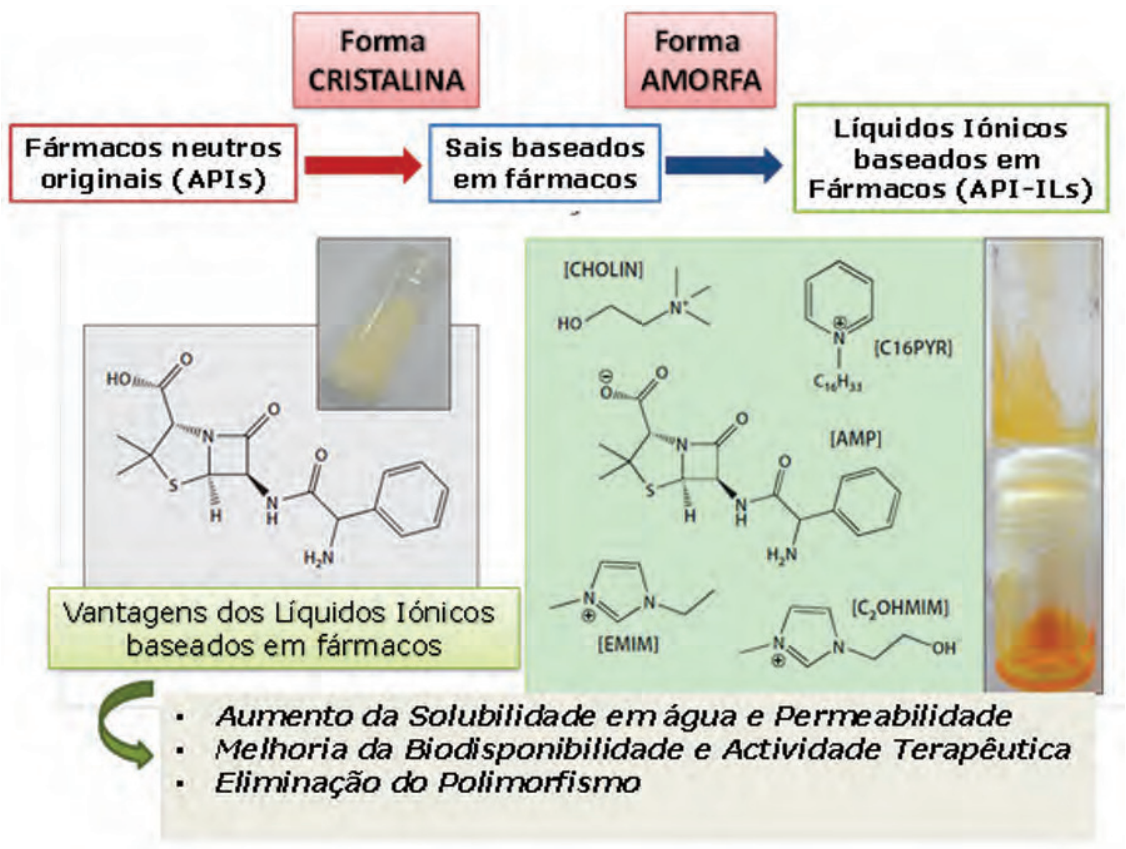

Figura 4 - Perspectivas futuras do uso de Líquidos Iónicos baseados em fármacos (API-ILs) obtidos a partir de fármacos neutros originais (APIs)

No caso dos LIs com anião ampicilina foi demonstrado um efeito de inibição e propriedades anti-bacterianas eficientes em especial em bactérias gram-negativas resistentes da Escherichia coli [34]. Alguns destes LIs também apresentaram uma elevada actividade antiproliferativa em estudos com linhas celulares humanas de cancro da próstata, cólon, mama e fígado [35].

O impacto dos novos LIs farmacêuticos no futuro da indústria farmacêutica está ainda por descobrir mas, por estarem na forma de sais em comparação com os princípios activos neutros, terão um muito maior potencial.

\section{AplicaÇõES EM ELECTROQUíMICA}

A larga janela electroquímica dos LIs tem atraído muitos investigadores a estudarem a sua utilização em diferentes campos da electroquímica. Diferentes tipos de LIs têm sido explorados em estudos de electroquímica em solução, processos de electrodeposição, electropolimerizações e utilização como electrólitos alternativos em semicondutores e baterias [36]. Recentemente, o uso de novos LIs como electrólitos eficientes em baterias, células de combustível e células solares tem sido também descrito e pode potenciar a utilização dos LIs em termos industriais [36]. Por outro lado têm sido apresentados exemplos do uso de LIs como electrólitos de sensores gasosos que detectam níveis de diferentes gases na ordem de ppm (em especial oxigénio, dióxido de carbono, amoníaco e dióxido de enxofre) [36].

Deve ser ainda realçado o contributo português no desenvolvimento de LIs electrocrómicos que possam funcionar em simultâneo como electrólito e electrocrómico em dispositivos reversíveis e eficientes [37]. Estes materiais electrocrómicos modificam as suas cores por acção da aplicação de um campo eléctrico [37]. Neste contexto foi possível preparar LIs baseados em aniões vanadato e complexos de cobalto-EDTA e mais recentemente diferentes catiões bipi- ridínio dissubstituídos [38]. De acordo com o componente electrocrómico é possível obter diferentes colorações e o respectivo contra-ião pode ser escolhido de modo a modelar algumas propriedades e aumentar a estabilidade e reversibilidade do sistema.

Outras aplicações dos LIs envolvem a sua utilização como meio reaccional e eletrólito de suporte em processos de electrossíntese e electrocatálise com possibilidade de reciclagem e facilidade na separação dos produtos finais [36]. Alguns LIs têm sido também utilizados em processos de electrodeposição de diferentes metais que normalmente se encontram limitados a sistemas aquosos [36].

\section{APLICAÇÕES EM QUÍMICA ANALITITICA}

O uso de LIs na área da química analítica tem sido explorada essencialmente em técnicas de separação e extracção. Diferentes estudos demonstram o potencial de utilização dos LIs para extracção e separação selectiva de compostos orgânicos e iões metálicos, tendo sido descritos exemplos de extracção líquido-líquido e micro-extracções de fase líquida e sólida [39].

Uma outra vertente de aplicação dos LIs relacionada com o seu uso em fases estacionárias para cromatografia gasosa apresenta-se largamente desenvolvida [40]. Diferentes LIs, incluindo quirais, têm sido desenvolvidos para competir com fases estacionárias para cromatografia gasosa. A elevada estabilidade térmica e eficiente capacidade de separação de compostos polares e não polares observada em diversos LIs podem contribuir para a sua aplicação em larga escala [40].

Na investigação de fases estacionárias quirais têm sido desenvolvidas misturas de derivados de ciclodextrinas em LIs bem como LIs quirais específicos como por exemplo ciclodextrinas incorporadas na estrutura de LIs [41]. Nas 
diferentes abordagens descritas tem sido possível manter a elevada estabilidade térmica e associar uma selectividade significativa para diferentes classes de compostos.

De destacar ainda o uso de LIs dissolvidos em acetonitrilo em electroforese capilar não aquosa, como electrólitos móveis para o revestimento capilar dinâmico de processos de electroforese de proteínas e como aditivos de eluição em cromatografia líquida [39].

Os LIs possuem uma elevada capacidade de solubilização e permitem absorver luz laser, podendo deste modo ser utilizados como matrizes de MALDI [39]. Os estudos de espectrometria de massa utilizando processos de ionização laser em matrizes assistidas (MALDI-MS) é um dos métodos de ionização mais eficazes na análise de biomoléculas polares e polímeros sintéticos de peso molecular elevado. Diferentes LIs têm sido testados como matrizes para análise de péptidos, proteínas, oligonucleótidos e açúcares. Em alguns casos, o uso de LIs como matrizes iónicas sólidas tem permitido uma significativa melhoria em termos de reprodutibilidade e sensibilidade para diversos sistemas [39].

Existem ainda exemplos de aplicação de LIs como potenciais solventes ou aditivos para ressonância magnética nuclear (RMN), permitindo o desenvolvimento de LIs deuterados como um desafio futuro a explorar [39].

\section{APLICAÇÕES EM ENGENHARIA QUÍMICA}

Nesta área os LIs têm sido muito estudados quer em termos da avaliação das suas propriedades fisico-químicas quer em processos de separação e extracção de compostos orgânicos e misturas de gases. De realçar o contributo português na utilização de membranas não porosas constituídas por superficies poliméricas hidrofóbicas ou hidrofílicas num processo de recuperação selectiva e quantitativa de diferentes solutos a partir de LIs [42]. Este processo designado por pervaporação permite efectuar uma separação não evaporativa e muito selectiva na recuperação de diferentes solutos voláteis de meios com líquido iónico [42]. Em paralelo têm sido desenvolvidas membranas poliméricas suportadas por LIs para o transporte selectivo de diferentes moléculas orgânicas com especial interesse na separação de aminas secundárias e terciárias [43] e membranas suportadas com LIs para processos de separação e purificação de gases em especial no tratamento de biometano e captura de $\mathrm{CO}_{2}$ a partir do gás de síntese [44].

Recentemente, LIs poliméricos têm sido utilizados em alternativa aos LIs convencionais devido a manterem as elevadas selectividades e permeabilidades e possuirem uma significativa estabilidade bem como propriedades mecânicas relevantes [45]. Nesse sentido diferentes polímeros de LIs (PILs) têm sido incorporados em membranas suportadas para processos de separação de gases tais como em misturas de dióxido de carbono com nitrogénio, metano e hidrogénio e ainda hidrogénio com nitrogénio [46].
Os LIs poliméricos apresentam-se como uma área de enorme potencial de aplicação não apenas em processos de separação mas também em áreas de ciência dos materiais e electroquímica [45].

A captura de $\mathrm{CO}_{2}$ é actualmente um tópico de interesse em que os LIs têm sido explorados [47]. Em 2005 foram descritos LIs reversíveis baseados na reacção de um álcool e de uma superbase orgânica (DBU) na presença de $\mathrm{CO}_{2}$ para a formação de carbonatos reversíveis [48]. A superbase fica protonada funcionando como catião combinado com o anião carbonato formado a partir do álcool. Este sal de carbonato pode ser convertido nos reagentes iniciais na presença de nitrogénio com libertação de $\mathrm{CO}_{2}$. Usando o mesmo conceito têm sido descritos outros estudos que incluem o uso de outras superbases orgânicas, aminas, aminoácidos e açucares em substituição de mono-álcoois [49].

No âmbito da engenharia química deve ainda ser referida a alargada investigação em sistemas bifásicos aquosos (ABS) e de líquido iónico com aplicação diversificada em separações e extracções selectivas [50]. A adição de agentes inorgânicos, sais orgânicos, aminoácidos e hidratos de carbono permitem uma alteração do equilíbrio de fases. A contribuição portuguesa neste tópico tem sido muito significativa com aplicação em processos de extracção de biomoléculas e outros compostos de valor acrescentado, remoção e recuperação de LIs de meios aquosos e na quebra de azeótropos [51].

Um dos projectos mais inovadores neste campo de investigação designa-se "IgYPurTech: uma tecnologia sustentável para a purificação de anticorpos”, e permitiu que a investigadora Doutora Mara Freire (CICECO, Univ. Aveiro) ganhasse uma bolsa ERC de 1,4 milhões de euros [52]. O objectivo do projecto será usar a imunoglobulina Y (IgY), um anticorpo produzido em grande quantidade e presente na gema de ovo usando técnicas não invasivas. Para o sucesso deste projecto será necessário desenvolver uma técnica de purificação eficaz que separe a imunoglobulina de outras proteínas contaminantes, de modo a que seja possível obter anticorpos com elevada pureza para a indústria farmacêutica e a um preço competitivo. O uso de sistemas aquosos bifásicos constituídos por LIs será o método a ser explorado neste projecto [52].

\section{ApLICAÇÕES EM OUTRAS ÁREAS CIENTÍFICAS}

Nos últimos anos, os LIs têm sido aplicados em diferentes áreas científicas com especial interesse na biotecnologia e bioquímica envolvendo processos de purificação de proteínas, isolamento de ácidos nucleicos e estudos detalhados de estabilização e interacção de LIs específicos com proteínas [53].

De destacar ainda o desenvolvimento de líquidos iónicos que possam funcionar como lubrificantes alternativos aos lubrificantes comerciais [54,55].

Os LIs magnéticos surgiram como uma classe de LIs de interesse através da incorporação de aniões paramagnéti- 
cos tais como ferro(III), manganês(II) e alguns lantanídeos [56]. Estes LIs magnéticos têm especial interesse para processos de catálise química e de extracção/separação podendo ser facilmente retirados através da aplicação dum campo magnético [57].

Na área da ciência dos materiais, os LIs têm surgido com especial interesse para dissolver diferentes materiais incluindo polímeros, estabilizar nanopartículas metálicas, combinar-se de forma eficiente com nanotubos de carbono e outros nanomateriais permitindo os seus estudos complementares e potenciando as suas aplicações em nanotecnologia $[58,59]$.

Uma das aplicações mais relevantes relaciona-se com o uso de LIs para a dissolução da celulose [60]. Depois de Graenacher em 1934 ter sugerido o uso de um sal fundido na dissolução de celulose, Rogers e colaboradores demonstraram em 2002 a possibilidade do uso de LIs baseados em aniões do tipo cloreto e acetato para a dissolução de elevadas quantidades de celulose [60].

Estudos recentes demonstram que a estrutura do anião coordenante e do catião são importantes no processo de solvatação [61]. Os catiões orgânicos com protões acídicos no anel heterocíclico (como o caso do catião imidazólio) permitem um aumento significativo da solubilidade devido à formação de ligações de hidrogénio com grupos hidroxilo e átomos de oxigénio do éter da celulose [61]. Actualmente os valores de solubilidade máxima para a celulose foram descritos para os LIs cloreto de 1-alil-3-metilimidazólio $\left(14,5 \mathrm{~m} / \mathrm{m} \%\right.$ a $\left.80^{\circ} \mathrm{C}\right)$ e acetato de 1-etil-3-metilimidazólio $\left(16 \mathrm{~m} / \mathrm{m} \%\right.$ a $90{ }^{\circ} \mathrm{C}$ e $25 \mathrm{~m} / \mathrm{m} \%$ com aquecimento de micro-ondas) [61]. Nos últimos anos diferentes derivados de celulose e outros biopolímeros têm sido estudados em combinação com LIs [60]. Por outro lado, a funcionalização da celulose tem sido possível em meio de líquido iónico permitindo a obtenção de diferentes derivados e biomateriais de elevado interesse comercial [61].

Com contributo português deve ser ainda realçado o desenvolvimento de novos líquidos iónicos funcionais intrinsicamente fotocrómicos [62], luminescentes [63], em estudos com compostos naturais (em especial flavílios) [64] e mais recentemente em aplicação para limpeza eficiente de resinas presentes em pinturas [65].

\section{Persectivas futuras}

Apesar do enorme crescimento da área dos LIs ao longo das últimas décadas, existe ainda uma margem de progressão alargada em especial em alguns campos de investigação. O contributo de diferentes áreas da química bem como da física, biologia, bioquímica, medicina e ciência dos materiais pode tornar a aplicação dos LIs ainda mais global e multidisciplinar. As propriedades peculiares dos LIs podem ser muito relevantes em processos de purificação de componentes biológicos mas também ser funcionalizados como materiais específicos de enorme potencial.
Nesse contexto, o uso de novos LIs incorporando moléculas biológicas e farmacêuticas pode contribuir significativamente para a química medicinal e biomédica. Os novos sais orgânicos farmacêuticos (API-ILs) têm um enorme potencial de aplicação na indústria farmacêutica tendo em atenção todos os estudos que já foram publicados.

Por outro lado, o desenvolvimento de novos polímeros de LIs (PILs) podem ter um impacto significativo em aplicações biológicas, em processos de separação de gases e em aplicações na energia e electrónica tais como células de combustível, solares, fotovoltaicas, baterias, termoacumuladores, entre outras.

O uso de LIs funcionais para processos de captura de $\mathrm{CO}_{2}$, processos químicos de catálise e nanocatálise e no processamento de produtos naturais de valor acrescentado como açúcares e biopolímeros devem ser tópicos relevantes a explorar nos próximos anos.

Nos últimos anos o aparecimento dos solventes eutécticos $[66,67]$ como uma subclasse dos LIs pode ter um interesse alargado em termos de estudo de propriedades e potenciais aplicações tendo por base a biocompatibilidade e o seu preço acessível para aplicações em larga escala.

A comunidade científica portuguesa deverá continuar a ter um destaque significativo na área dos LIs tendo por base a multidisciplinaridade dos grupos de investigação envolvidos e a interligação com centros de investigação internacionais de excelência.

O maior desafio para toda a comunidade dos LIs prende-se com a capacidade de conseguirem uma transferência tecnológica entre a investigação académica e as suas potenciais aplicações industriais.

\section{REFERÊNCIAS}

[1] A.Kokorin, Ionic Liquids: Theory, Properties, New Approaches, Intech, 2011

[2] P.Walden, Bull. Acad. Imper. Sci. (1914) 1800

[3] F.H.Hurley, T.P.Wier, J. Electrochem. Soc. 98 (1951) 203$-206$

[4] M.Freemantle, An Introduction to Ionic Liquids, RSC Publishing, 2009

[5] M.J. Earle, J.M.S.S. Esperança, M.A. Gilea, J.N. Canongia Lopes, L.P.N. Rebelo, J.W. Magee, K.R. Seddon, J.A. Widegren, Nature 439 (2006) 831-834

[6] N.V. Plechkova, K.R. Seddon, Chem. Soc. Rev. 37 (2008) 123-150

[7] M. Freemantle, Chem. Eng. News 81 (2003) 9

[8] P. Wassercheid, T. Welton, Ionic Liquids in synthesis, Wiley-VCH, 2007

[9] C. Hardacre, V. Parvulescu (Eds), Catalysis in Ionic Liquids: From catalyst synthesis to Application, RSC Publishing, 2014 
[10] J.P. Hallett, T. Welton, Chem. Rev. 111 (2011) 3508-3576

[11] D. Cole-Hamilton, R. Tooze (Eds), Catalyst Separation, Recovery and Recycling, Springer, 2006

[12] S. Keskin, D. Kayrak-Talay, U. Akuran, O. Hortaçsu, J. Supercritical Fluids 43 (2007) 150-180

[13] R. Fehrmann, A. Riisager, M. Haumann (Eds), Supported Ionic Liquids: Fundamentals and Applications, WileyVCH, 2014

[14] T. Payagala, D. W. Armstrong, Chirality 24 (2012) 17-53

[15] K. Zalewska, L.C. Branco, Mini-Reviews Org. Chem. 11 (2014) 141-153

[16] M. Prechtl, J.D. Scholten, B.A.D. Neto, J. Dupont, Current Org. Chem. 13 (2009) 1259-1277

[17] S. Luo, X. Hi, L. Zhang, S. Liu, H. Xu, J.P. Cheng, Angew. Chem. Int. Ed. 45 (2006) 3093-3097

[18] L.C. Branco, A. Serbanovic, M.N. da Ponte, C.A.M. Afonso, ACS Catalysis 1 (2011) 1408-1413

[19] L. Poletti, C. Chiappe, L. Lay, D. Pieraccini, L. Polito, G. Russo, Green Chem. 9 (2007) 337-341

[20] F.V. Rautwijk, R. Sheldon, Chem. Rev. 107 (2007) 2757$-2785$

[21] P. Lozano, T. De Diego, J.L. Iborra (Eds), Biocatalytic Processes using Ionic Liquids and supercritical carbon dioxide, VCH-Wiley, 2010

[22] F. Liu, L. Wang, Q. Sun, L. Zhu, X. Meng, F.S. Xiao, J. Am. Chem. Soc. 134 (2012) 16948-16950

[23] N.M.T. Lourenço, C.A.M. Afonso, Angew. Chem. Int. Ed. 46 (2007) 8178-8181

[24] I.M. Marrucho, L.C. Branco, L.P.N. Rebelo, Annu. Rev. Chem. Biomol. Eng. 8 (2014) 527-546

[25] K.J. Kulacki, G.A. Lamberti, Green Chem. 10 (2008) 104$-110$

[26] R.F.M. Frade, A.A. Rosatella, C.S. Marques, L.C. Branco, P.S. Kulkarni, N.M.M. Mateus, C.A.M. Afonso, C.M.M. Duarte, Green Chem. 11 (2009) 1660-1665

[27] M. Petkovic, J.L. Ferguson, M.Q. Gunaratne, R. Ferreira, M.C. Leitão, K.R. Seddon, L.P.N. Rebelo, C.S. Pereira, Green Chem. 12 (2010) 643-649

[28] M.T. Viciosa, G.Santos, A.Costa, F.Danède, L.C. Branco, N.Jordão, N.T. Correia, M. Dionisio, Phys. Chem. Chem. Phys. 17 (2015) 24108-24120

[29] R. Ferraz, L.C. Branco, C. Prudêncio, J.P. Noronha, Z. Petrovski, ChemMedChem 6 (2011) 975-985

[30] W.L. Hough, M. Smiglak, H. Rodríguez, R.P. Swatloski, S.K. Spear, D.T. Daly, J. Pernak, J.E. Grisel, R.D. Carliss, M.D. Soutullo, J.H. Davis, R.D. Rogers, New. J. Chem. 31 (2007) 1429-1436

[31] R. Ferraz, L.C. Branco, I.M. Marrucho, J.M.M. Araújo, M.N. da Ponte, C. Prudêncio, J.P. Noronha, Z. Petrovski, Med. Chem. Commun. 3 (2012) 494-497

[32] C. Florindo, J.M.M. Araújo, F. Alves, C. Matos, R. Ferraz, C. Prudêncio, J.P. Noronha, Z. Petrovski, L. Branco, L.P.N.
Rebelo, I.M. Marrucho, Int. J. of Pharmaceutics 456 (2013) 553-559

[33] C. Florindo, A. Costa, C. Matos, S.L. Nunes, A.N. Matias, C.M.M. Duarte, L.P.N. Rebelo, L.C. Branco, I.M. Marrucho, Int. J. of Pharmaceutics 469 (2014) 179-189

[34] R. Ferraz, V. Teixeira, D. Rodrigues, R. Fernandes, C. Prudêncio, J.P. Noronha, Z. Petrovski, L.C. Branco, RSC Adv. 4 (2014) 4301-4307

[35] R. Ferraz, J.C. Rodrigues, M.H. Fernandes, M.M. Santos, I.M. Marrucho, L.P. N. Rebelo, C. Prudêncio, J.P. Noronha, Z. Petrovski, L.C. Branco, ChemMedChem 10 (2015) 1480-1483

[36] H. Ohno, Electrochemical Aspects of Ionic Liquids, Wiley, 2011

[37] A. Branco, L.C. Branco, F. Pina, Chem. Commun. 47 (2011) 2300-2302

[38] N. Jordão, L. Cabrita, F. Pina, L.C. Branco, Chem. Eur. J. 20 (2014) 3982-3988

[39] R.J. S. Hein, M.M. Waruke, D.W. Armstrong, Annu. Rev. Anal. Chem. 2 (2009) 145-168

[40] C.F. Poole, S.K. Poole, J. Sep. Science, 34 (2011) 888-900

[41] N. Costa, S. Matos, M.D.R.G. Silva, M.M.A. Pereira, ChemPlusChem 78 (2013) 1466-1474

[42] T. Schafer, C.M. Rodrigues, C.A.M. Afonso, J.G. Crespo, Chem. Commun. (2001) 1622-1623

[43] L.C. Branco, J.G. Crespo, C.A.M. Afonso, Angew. Chem. Int. Ed. 41 (2002) 2771-2773

[44] H. Karkhanechi, S. Salmani, M. Asghari, ChemBioEng Reviews 2 (2015) 290-302

[45] S. Zulfigar, M. I. Sarwar, D. Mecerreyes, Polym. Chem. 6 (2015), 6435-6451.

[46] L.C. Tomé, M.A. Aboudzadeh, L.P.N. Rebelo, C.S.R. Freire, D. Mecerreyes, I.M. Marrucho, J. Mater. Chem. A 1 (2013), 10403-10411

[47] E.D. Bates, R.D. Mayton, I. Ntai, J.H. Davis, J. Am. Chem. Soc 124 (2002) 926-927

[48] P.G. Jessop, D.J. Heldebran, C.A. Eckert, C.L. Liotta, Nature 436 (2005) 1102

[49] G.V.S.M. Carrera, N. Jordão, M.M. Santos, M.N. da Ponte, L.C. Branco, RSC Advances 5 (2015), 35564-35571

[50] M.G. Freire, A.F.M. Claúdio, J.M.M. Araujo, J.A.P. Coutinho, I.M. Marrucho, J.N.C. Lopes, L.P.N. Rebelo, Chem. Soc. Rev. 41 (2012) 4966-4995

[51] A. B. Pereiro, J.M.M. Araujo, J.M.S.S. Esperança, I.M. Marrucho, L.P.N. Rebelo, J. Chem. Thermodynamics 46 (2011) 2-28

[52] M. Taha, M.R. Almeida, F.A. Silva, P. Domingues, S.P.M. Ventura, J.A.P. Coutinho, M.G. Freire, Chem. Eur. J. 21 (2015) 4781-4788

[53] A.M. Figueiredo, J. Sardinha, G.R. Moora, E.J. Cabrita, Phys. Chem. Chem. Phys. 15 (2013) 19632-19643

[54] F. Zhou, Y. Liang, W. Liu, Chem. Soc. Rev. 38 (2009) 2590$-2599$ 
[55] G. Tiago, J. Restolho, A. Forte, R. Colaço, L.C. Branco, B. Saramago, Colloids Surf. A 472 (2015) 1-8

[56] E. Santos, J. Albo, A. Irabieu, RSC Adv. 4 (2014) 40008$-40018$

[57] S.K. Panja, S. Saha, RSC Adv. 3 (2013) 14495-14500

[58] Z. He, P. Alexandridis, Phys. Chem. Chem. Phys. 17 (2015) 18238-18261

[59] M. Tunckol, J. Durand, P. Serp, Carbon 50 (2012) 4303-4334

[60] R.P. Swatloski, S.K. Spear, J.D. Holbrey, R.D. Rogers, J. Am. Chem. Soc. 124 (2002) 4974-4975

[61] H. Wang, G. Gurau, R.D. Rogers, Chem. Soc. Rev. 41 (2012) 1519-1537
[62] L.C. Branco, F. Pina, Chem. Comm. (2009) 6204-6206

[63] J. Delgado, M. Vilarigues, L.C. Branco, C.A.T. Laia, Chem. Eur. J. 21 (2015) 726-732

[64] F. Pina, J.C. Lima, A.J. Parola, C.A.M. Afonso, Angew. Chem. Int. Ed. 43 (2004) 1525-1527

[65] M.F. Pacheco, A.I. Pereira, L.C. Branco, A.J. Parola, J. Material Chem. A 1 (2013) 7016-7018

[66] E.L. Smith, A.P. Abbott, K.S. Ryder, Chem. Rev. 114 (2014) 11060-11082

[67] A. Paiva, R. Craveiro, I. Aroso, M. Martins, R.L. Reis, A.R.C. Duarte, ACS Sust. Chem. Eng. 2 (2014) 1063$-1071$

\section{Actualidades CientíFICAS}

\section{LÍQUDOS COM POROSIDADE PERMANENTE}

Um grupo de investigadores produziu um conjunto de líquidos com porosidade permanente. Esta nova classe de materiais porosos, que combina os benefícios de um líquido com os de um sólido adsorvente, pode encontrar aplicações em áreas importantes, tais como a catálise ou a captura de carbono.

Sólidos porosos, tais como zeólitos ou MOFs, são materiais estruturalmente rígidos que possuem cavidades com formas e tamanhos regulares que os tornam, por exemplo, bastante uteis em processos de separação e catálise. Por outro lado, a "porosidade” em líquidos convencionais está limitada a cavidades mal definidas e apenas temporárias entre suas moléculas.

Apesar das suas inúmeras e importantes aplicações, a natureza sólida dos zeólitos e MOFs impõe algumas limitações, nomeadamente na implementação em processos de fluxo convencionais. Assim, materiais que combinem a fluidez característica dos líquidos e a porosidade permanente característica dos sólidos são de grande interesse tecnológico.

Uma equipa internacional de investigadores (Reino Unido, França, Alemanha e Argentina), preparou recentemente um conjunto de líquidos com porosidade permanentemente baseados em éteres de coroa. Os sistemas obtidos, do tipo "gaiola", têm a vantagem de poderem ser dissolvidos, servindo assim como unidades porosas solúveis. Os testes realizados mostraram que foi possível aumentar bastante a solubilidade do metano com estes líquidos. Os estudos realizados podem fornecer a base para o desenvolvimento de uma nova classe de materiais porosos funcionais, nomeadamente para aplicação em catálise e separação, captura e transporte de gases.

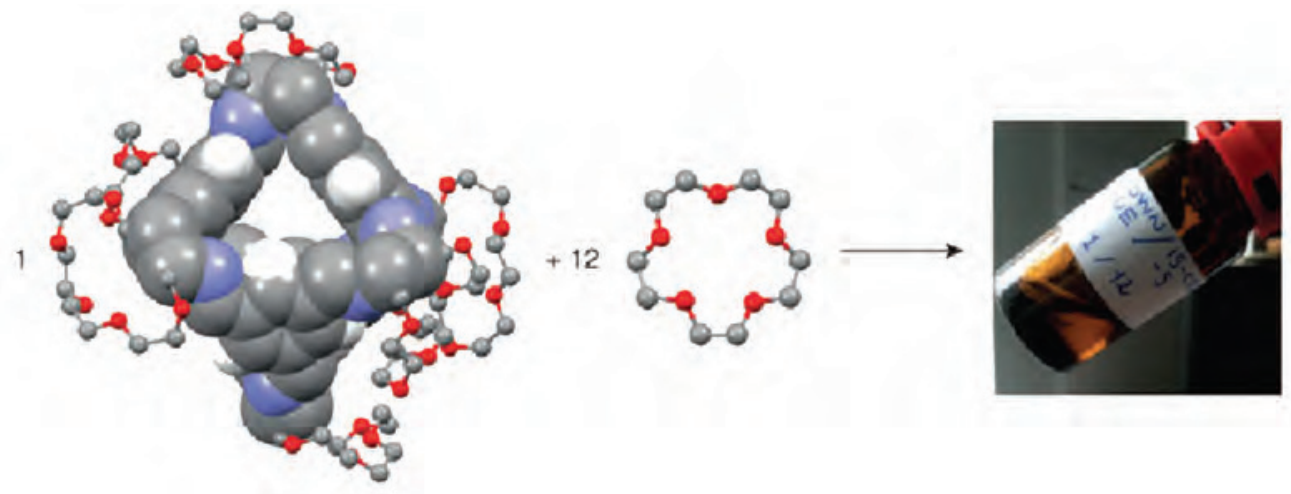

(Fontes: First permanently porous liquid created, http://www.rsc.org/chemistryworld/2015/11/first-permanentlyporous-liquids-created e N. Giri, M.G. Del Pópolo, G. Melaugh, R.L. Greenaway, K. Rätzke, T. Koschine, L. Pison,

M.F.C. Gomes, A.I. Cooper, S.L. James. Liquids with permanent porosity. Nature (2015) DOI: 10.1038/nature16072)

Paulo Mendes (pjgm@uevora.pt) 